\title{
Sharp lower bounds on expectations of gOS based on DGFR distributions
}

\author{
Mariusz Bieniek $^{1}$ (D) Agnieszka Goroncy $^{2}$ (D)
}

Received: 24 April 2017 / Revised: 24 November 2017 / Published online: 4 December 2017

(C) The Author(s) 2017. This article is an open access publication

\begin{abstract}
We present the lower bounds expressed in standard deviation units on the expectations of the generalized order statistics (gOS) which are based on the parent distributions with the decreasing generalized failure rate. The particular cases are families of distributions with the decreasing density and decreasing failure rate. The bounds are obtained with the use of the projection method applied to functions satisfying some particular conditions and appropriately chosen convex cones. We also provide the attainability conditions. The results are illustrated with the numerical results on the progressively type II censored order statistics, which are one of the special cases of $\mathrm{gOS}$.
\end{abstract}

Keywords Bound - Generalized order statistics · Convex transform order · Decreasing generalized failure rate $\cdot$ Decreasing density $\cdot$ Decreasing failure rate

Mathematics Subject Classification 60E15 · 62G32

Agnieszka Goroncy

gemini@mat.umk.pl

Mariusz Bieniek

mariusz.bieniek@umcs.lublin.pl

1 Institute of Mathematics, University of Maria Curie-Skłodowska, Pl. M. Curie-Skłodowskiej 1, 20-031 Lublin, Poland

2 Nicolaus Copernicus University, Chopina 12/18, 87-100, Toruń, Poland 


\section{Introduction}

Assume that $F$ is arbitrary distribution with the quantile function

$$
F^{-1}(u)=\sup \{x \in \mathbb{R}: F(x) \leq u\}, \quad u \in[0,1),
$$

and finite moments

$$
\mu=\int_{0}^{1} F^{-1}(x) d x, \quad \sigma^{2}=\int_{0}^{1}\left(F^{-1}(x)-\mu\right)^{2} d x .
$$

Let $\gamma=\left(\gamma_{1}, \ldots, \gamma_{n}\right)$ be the vector of positive numbers. The generalized order statistics (gOSs, for short) $X_{\gamma}^{(1)}, \ldots, X_{\gamma}^{(n)}$ based on $F$ with the parameters $\gamma$ were introduced by Kamps $(1995 \mathrm{a}, \mathrm{b})$ by means of the quantile transformation

$$
X_{\gamma}^{(r)}=F^{-1}\left(U_{\gamma}^{(r)}\right), \quad r=1, \ldots, n,
$$

where $U_{\gamma}^{(1)}, \ldots, U_{\gamma}^{(n)}$ denote the uniform generalized order statistics with the joint density function of the form

$$
f_{U_{\gamma}^{(1)}, \ldots, U_{\gamma}^{(n)}}\left(u_{1}, \ldots, u_{n}\right)=k\left(\prod_{j=1}^{n-1} \gamma_{j}\right)\left(\prod_{i=1}^{n-1}\left(1-u_{i}\right)^{m_{i}}\right)\left(1-u_{n}\right)^{k-1},
$$

for $0 \leq u_{1} \leq \cdots \leq u_{n}<1$, where $m_{i}=\gamma_{i}-\gamma_{i+1}-1$ for all $i=1, \ldots, n-1$ and $k=\gamma_{n}$.

The model of gOSs contains various models of ordered statistical data as special cases. For instance, if $\gamma_{r}=n-r+1,1 \leq r \leq n$, then gOSs coincide in the distributional sense with ordinary order statistics of the random sample of size $n$. Other special cases include various models of record values, sequential order statistics or progressively type II order statistics. For details the reader is referred, e.g., to Cramer and Kamps (2003). The main role of the model is to unify the study of various models of ordered random variables, and to provide a unified approach to a broad variety of problems. Examples of such approach may be found for instance in the papers by Bieniek (2006, 2008a, b), Cramer et al. (2002, 2004), Rychlik (2010) and Goroncy (2014) (optimal bounds on expectations of gOS), Belzunce and Martínez-Riquelme (2015) (stochastic ordering of gOSs), Mahmoud and Al-Nagar (2009) (recurrence relations on moments of gOSs and related characterizations of distributions) and Barakat et al. (2016) (prediction of future values of gOSs).

To formulate the main aim of this paper we need to introduce the following notations and definitions. For a fixed $\alpha \in \mathbb{R}$ the generalized Pareto distributions (GPDs) are defined as follows 


$$
W_{\alpha}(x)=\left\{\begin{array}{lll}
1-(1-\alpha x)^{1 / \alpha}, & \text { for } x \geq 0 & \text { if } \alpha<0 \\
1-(1-\alpha x)^{1 / \alpha}, & \text { for } 0 \leq x \leq \frac{1}{\alpha} & \text { if } \alpha>0 \\
1-\mathrm{e}^{-x}, & \text { for } x \geq 0 & \text { if } \alpha=0
\end{array}\right.
$$

Consider the distribution function $F$ which succeeds the GPD distribution in the convex transform order, i.e., $F \succ_{c} W_{\alpha}$, which means that the composition $F^{-1} W_{\alpha}$ is a convex function on the support of $W_{\alpha}$. This condition is equivalent to $W_{\alpha}^{-1} F$ being concave on the support of $F$ and if $F$ is absolutely continuous with the density function $f$, the derivative

$$
\left(W_{\alpha}^{-1} F\right)^{\prime}(y)=(1-F(y))^{\alpha-1} f(y)
$$

is decreasing. Bieniek (2008a) introduced the family of distributions with the decreasing generalized failure rate (DGFR) as follows

$$
\operatorname{DGFR}(\alpha)=\left\{F: F \succ_{c} W_{\alpha}\right\},
$$

(see also Bieniek 2007a) with the generalized failure rate of an absolutely continuous $F$ defined as

$$
\gamma_{\alpha}(y)=(1-F(y))^{\alpha-1} f(y),
$$

(see Barlow and van Zwet 1970). Note that if $\alpha=1$, then $W_{1}$ becomes the standard uniform distribution, and if $\alpha=0$, then $W_{0}$ is the standard exponential distribution, hence obviously, DGFR(0) $=$ DFR and DGFR(1) $=$ DD.

The problem we consider in this paper is to find the lower bounds on the expectations of the standardized generalized order statistics

$$
\mathbb{E} \frac{X_{\gamma}^{(r)}-\mu}{\sigma},
$$

when the underlying distribution function $F$ belongs to the family of distributions with the decreasing generalized failure rate (DGFR). The issue of the bounds on the expectations of gOSs has been extensively examined recently, beginning with the general case considered by Cramer et al. (2002), who presented the upper nonnegative bounds, expressed in scale units generated by absolute central moments of various orders, using the projection method (for details see Rychlik 2001). Later, Goroncy (2014) completed these results by establishing the upper non-positive and all lower bounds on (1) for an arbitrary distribution function $F$, considering also the case of bounds expressed in the absolute central moments units. In general, lower negative (upper positive) and lower positive (upper negative) bounds are obtained by use of different methods.

Non-positive lower bounds on expectations of gOSs in the general case of the baseline distribution function $F$ were derived by use of the projection method, based on the greatest convex minorants (see Moriguti 1953). Below we briefly describe the procedure. 
First, we make use of the representation due to Cramer and Kamps (2003) of the probability density function of the $r$ th uniform $\operatorname{gOS} U_{\gamma}^{(r)}$ in the form

$$
\begin{aligned}
f_{*, r}(x) & =c_{r-1} G_{r}\left(x \mid \gamma_{1}, \ldots, \gamma_{r}\right) \\
& =c_{r-1} \mathrm{G}_{r, r}^{r, 0}\left(1-x \mid \begin{array}{c}
\gamma_{1}, \ldots, \gamma_{r} \\
\gamma_{1}-1, \ldots, \gamma_{r}-1
\end{array}\right), \quad x \in(0,1),
\end{aligned}
$$

where $c_{r-1}=\prod_{j=1}^{r} \gamma_{j}$ and $\mathrm{G}_{r, r}^{r, 0}$ is a particular Meijer's G-function defined by

$$
\mathrm{G}_{r, r}^{r, 0}\left(\begin{array}{l}
s \mid \begin{array}{c}
\gamma_{1}, \ldots, \gamma_{r} \\
\gamma_{1}-1, \ldots, \gamma_{r}-1
\end{array}
\end{array}\right)=\frac{1}{2 \pi i} \int_{L} \frac{s^{z}}{\prod_{j=1}^{r}\left(\gamma_{j}-1-z\right)} d z
$$

and $L$ is appropriately chosen contour of integration. For the definition of a general G-function see Chaps. 2 and 3 of Mathai (1993). Note that (2) implies that changing the order of $\gamma_{1}, \ldots, \gamma_{r}$ does not affect $f_{*, r}$ and therefore without loss of generality we may and do assume that $\gamma_{1} \geq \ldots \geq \gamma_{r}>0$. Moreover, we denote by $F_{*, r}$ the cumulative distribution function of $U_{\gamma}^{(r)}$.

Secondly, the expectation of the $r$ th gOS is given by

$$
\mathbb{E} X_{\gamma}^{(r)}=\int_{0}^{1} F^{-1}(u) f_{*, r}(u) d u
$$

Denoting $h_{*, r}=1-f_{*, r}$, and noting that $h_{*, r}$ integrates to 0 over $[0,1]$ we have

$$
-\left(\mathbb{E} X_{\gamma}^{(r)}-\mu\right)=\int_{0}^{1}\left(F^{-1}(u)-\mu\right) h_{*, r}(u) d u .
$$

Consider the convex cones

$$
\begin{aligned}
\mathscr{C} & =\left\{g \in L^{2}(0,1): g \text { is nondecreasing }\right\} \\
\mathscr{C}^{0} & =\left\{g \in \mathscr{C}: \int_{0}^{1} g(u) d u=0\right\}
\end{aligned}
$$

in the usual Hilbert space $L^{2}(0,1)$ of square integrable functions on $[0,1]$. Denote by $P$ and $P^{0}$ the projection operators onto $\mathscr{C}$ and $\mathscr{C}^{0}$, respectively. Then we have

$$
-\left(\mathbb{E} X_{\gamma}^{(r)}-\mu\right) \leq \int_{0}^{1}\left(F^{-1}(u)-\mu\right) P^{0} h_{*, r}(u) d u .
$$

Since the cone $\mathscr{C}$ is translation invariant then

$$
\int_{0}^{1} P h_{*, r}(u) d u=\int_{0}^{1} h_{*, r}(u) d u=0
$$


Therefore $P h_{*, r} \in \mathscr{C}^{0}$ and $P^{0} h_{*, r}=P h_{*, r}$. Using the Schwarz inequality we obtain the following bound

$$
\frac{\mathbb{E} X_{\gamma}^{(r)}-\mu}{\sigma} \geq-\left\|P h_{*, r}\right\|
$$

The projection $P h_{*, r}$ is determined using the greatest convex minorant method of Moriguti (1953). Cramer et al. (2004) have shown that $f_{*, r}$ is a unimodal function (see also Bieniek 2007b). Accordingly, assuming that $H_{*, r}$ denotes the antiderivative of $h_{*, r}$ we need to determine $\delta$ such that $H_{*, r}(\delta)=\delta h_{*, r}(\delta)$, or equivalently,

$$
F_{*, r}(\delta)=\delta f_{*, r}(\delta)
$$

hence

$$
P h_{*, r}(u)= \begin{cases}1-f_{*, r}(\delta), & \text { for } 0 \leq u \leq \delta \\ 1-f_{*, r}(u), & \text { for } \delta<u \leq 1 .\end{cases}
$$

Therefore we have the following bound

$$
\frac{\mathbb{E} X_{\gamma}^{(r)}-\mu}{\sigma} \geq-\left(\delta\left(f_{*, r}(\delta)\right)^{2}+\int_{\delta}^{1}\left(f_{*, r}(u)\right)^{2} d u-1\right)^{1 / 2} .
$$

The equality holds for the distribution function satisfying the following condition

$$
\frac{F^{-1}(u)-\mu}{\sigma}=\frac{P h_{*, r}(u)}{\left\|P h_{*, r}\right\|}, \quad u \in(0,1) .
$$

Note that the Moriguti (1953) approach has been also used, e.g., by Okolewski and Kaluszka (2008) to derive sharp upper bounds on the expectations of concomitants of order statistics or by Bieniek (2016b) to derive optimal bounds on the bias of trimean.

\section{Auxiliary results}

In this paper we are interested in establishing the lower negative bounds on the expectations of the gOSs based on the DGFR families of distributions. Therefore we need to adapt the procedure described in the previous section, imposing additional restrictions on $F$. The upper bounds in the restricted families of DD, DDA and DFR, DFRA distributions have been already considered by Bieniek (2006, 2008b), who established the positive projection bounds, and recently by Goroncy (2017), who examined the negative ones in the DD and DDA families.

Fix a distribution function $W$ on the interval $[0, d)$, where $d \leq \infty$, with the probability density function $w$ such that

$$
\int_{0}^{d} x^{2} w(x) d x<\infty
$$


We consider the Hilbert space $\mathscr{L}_{W}^{2}$ of square integrable functions with respect to the weight $w$, and we define the following convex cones in $\mathscr{L}_{W}^{2}$

$$
\begin{aligned}
& \mathscr{C}_{W}=\left\{g \in \mathscr{L}_{W}^{2}: g \text { is nondecreasing and convex }\right\}, \\
& \mathscr{C}_{W}^{0}=\left\{g \in \mathscr{C}_{W}: \int_{0}^{d} g(u) w(u) d u=0\right\} .
\end{aligned}
$$

By $P_{W}$ and $P_{W}^{0}$ we denote the projection operators onto $\mathscr{C}_{W}$ and $\mathscr{C}_{W}^{0}$, respectively. In what follows we use the notation $\hat{f}_{*, r}=f_{*, r} \circ W$ and $\hat{h}_{*, r}=h_{*, r} \circ W$. Changing the variables in (3) we have

$$
\begin{aligned}
-\left(\mathbb{E} X_{\gamma}^{(r)}-\mu\right) & =\int_{0}^{d}\left(F^{-1} W(u)-\mu\right) \hat{h}_{*, r}(u) w(u) d u \\
& \leq \int_{0}^{d}\left(F^{-1} W(u)-\mu\right) P_{W}^{0} \hat{h}_{*, r}(u) w(u) d u .
\end{aligned}
$$

Taking into account that

$$
\int_{0}^{d} \hat{h}_{*, r}(u) w(u) d u=\int_{0}^{1} h_{*, r}(t) d t=0,
$$

and appealing to the translation invariance of $C_{W}$, similar arguments as in the previous section prove that $P_{W}^{0} \hat{h}_{*, r}=P_{W} \hat{h}_{*, r}$. Therefore, using (7), we have

$$
\frac{\mathbb{E} X_{\gamma}^{(r)}-\mu}{\sigma} \geq-\left\|P_{W} \hat{h}_{*, r}\right\|_{W}
$$

where for an arbitrary $f \in \mathscr{L}_{W}^{2}$ we denote the $\mathscr{L}_{W}^{2}$-norm of $f$ by

$$
\|f\|_{W}=\left(\int_{0}^{d}|f(u)|^{2} w(u) d u\right)^{1 / 2} .
$$

The equality in (8) is attained for distribution functions $F$ satisfying the following condition:

$$
\frac{F^{-1} W(u)-\mu}{\sigma}=\frac{P_{W} \hat{h}_{*, r}(u)}{\left\|P_{W} \hat{h}_{*, r}\right\|_{W}}, \quad u \in(0,1) .
$$

\subsection{The shapes of the projected functions}

In order to determine $P_{W} \hat{h}_{*, r}$ it is crucial to study the monotonicity and convexity properties of the functions $\hat{f}_{*, r}$ and $\hat{h}_{*, r}$. The shape of $\hat{f}_{*, r}$ was briefly described by Bieniek (2008a), but here we need much more precise statement. This requires the 
knowledge of sign changes of the derivatives $\hat{f}_{*, r}^{\prime}$ and $\hat{f}_{*, r}^{\prime \prime}$. (Bieniek 2008a, Lemma 2.1) argued that these properties can be studied effectively only if $W$ satisfies the condition $W^{\prime \prime}(x)(1-W(x))=c\left(W^{\prime}(x)\right)^{2}$, i.e., when $W=W_{\alpha}$ for some $\alpha \in \mathbb{R}$. Then we may apply the following variation diminishing property (VDP) of the functions $\hat{f}_{*, 1}, \ldots, \hat{f}_{*, r}$, which is an easy consequence of Theorems 1,2 and 3 of Bieniek (2007b).

Proposition 1 (VDP) Assume that $r \in \mathbb{N}$ and $\gamma_{1} \geq \gamma_{2} \geq \cdots \geq \gamma_{r}>0$. Then the number of zeroes in $(0, d)$ of any linear combination $\sum_{j=1}^{r} a_{j}{\hat{f_{*, j}}}_{\text {in }}$ the support of $W_{\alpha}$ does not exceed the number of sign changes in the sequence $\left(a_{1}, \ldots, a_{r}\right)$ of its coefficients. Moreover, the first and the last signs of the combination are the same as the signs of the first and last non zero elements of $\left(a_{1}, \ldots, a_{r}\right)$, respectively.

Next we recall the expressions for the first and second derivatives of $\hat{f}_{*, r}=f_{*, r} W_{\alpha}$ obtained by Bieniek (2008a)

$$
\hat{f}_{*, r}^{\prime}(x)=\frac{1}{1-\alpha x}\left(\gamma_{r} \hat{f}_{*, r-1}(x)-\left(\gamma_{r}-1\right) \hat{f}_{*, r}(x)\right),
$$

and

$$
\hat{f}_{*, r}^{\prime \prime}(x)=\frac{1}{(1-\alpha x)^{2}}\left[a_{r-2} \hat{f}_{*, r-2}(x)+a_{r-1} \hat{f}_{*, r-1}(x)+a_{r} \hat{f}_{*, r}(x)\right],
$$

where

$$
\begin{aligned}
a_{r-2} & =\gamma_{r-1} \gamma_{r}>0 \text { for } r \geq 3, \\
a_{r-1} & =-\gamma_{r}\left(\gamma_{r}+\gamma_{r-1}-\alpha-2\right), \\
a_{r} & =\left(\gamma_{r}-1\right)\left(\gamma_{r}-\alpha-1\right) .
\end{aligned}
$$

The above expressions for $\hat{f}_{*, r}^{\prime}$ and $\hat{f}_{*, r}^{\prime \prime}$ are valid also for $r=1$ and $r=2$ if we adopt the convention $\hat{f}_{*, 0}=\hat{f}_{*,-1} \equiv 0$.

Recall that by Lemma 2.2 of Cramer et al. (2004) we have the following values of $\hat{f}_{*, r}$ at 0 and $d$

$\hat{f}_{*, r}(0)=\left\{\begin{array}{ll}\gamma_{1}, & \text { if } r=1, \\ 0, & \text { if } r \geq 2,\end{array} \quad \lim _{x \rightarrow d} \hat{f}_{*, r}(x)= \begin{cases}0, & \text { if } \gamma_{r}>1, \\ A>1, & \text { if } \gamma_{r-1}>\gamma_{r}=1, \\ +\infty, & \text { if } \gamma_{r-1}=\gamma_{r}=1 \text { or } \gamma_{r}<1 .\end{cases}\right.$

Next, using (10) and VDP it is easy to see that each $\hat{f}_{*, r}, r \geq 2$, is increasingdecreasing if $\gamma_{r}>1$ or increasing if $\gamma_{r} \leq 1$. Moreover we note that since $\hat{f}_{*, r}$ integrates to 1 with weight $w_{\alpha}$ over the support of $W_{\alpha}$, then in any case the maximum of $\hat{f}_{*, r}$ is greater than 1 . Summarizing the above considerations we easily determine the shape of $\hat{f}_{*, r}$ which is described in the following lemma.

Lemma 1 The shape of $\hat{f}_{*, r}=f_{*, r} W_{\alpha}$ for $r \geq 2$ is as follows: 
(i) If $\gamma_{r} \leq 1$, then $\hat{f}_{*, r}$ is strictly increasing on $(0, d)$ from 0 to either $A>1$ or $A=+\infty$;

(ii) If $\alpha>0$ and $\gamma_{r} \in(1,1+\alpha]$, then there exists $a \in(0, d)$ such that $\hat{f}_{*, r}$ is increasing on $(0, a)$ from 0 to $\hat{f}_{*, r}(a)>1$ and then concave decreasing on $(a, d)$ to 0

(iii) If $\gamma_{r}>1+\max (\alpha, 0)$, then there exist $a, b \in(0, d)$ such that $a<b$ and $\hat{f}_{*, r}$ is increasing on $(0, a)$ from 0 to $\hat{f}_{*, r}(a)>1$, then concave decreasing on $(a, b)$, and finally convex decreasing on $(b, d)$ to 0 .

Obviously, the convexity properties of $\hat{f}_{*, r}$ on $(0, a)$ may also be established, but as it turns out later they are irrelevant for our purposes.

\subsection{The results on the projection problem}

The projection of $\hat{h}_{*, r}$ onto $\mathscr{C}_{W}$ will be determined using the following extension of the results of Danielak and Rychlik (2004). We consider the class of functions $h:[0, d) \rightarrow \mathbb{R}$ satisfying the following set of conditions:

(A) $h$ is a bounded, twice differentiable function, such that $h(0) \geq 0, h(d):=$ $\lim _{x \nearrow d} h(x) \geq 0$ and $\int_{0}^{d} h(x) w(x) d x=0$, where $w$ is a positive weight function satisfying $\int_{0}^{d} w(x) d x=1$. Moreover, $h$ is decreasing on $(0, a)$, convex increasing on $(a, b)$, and concave increasing on $(b, d)$, for some $0<a<b<d$.

This set of conditions is the same as the one considered by Danielak and Rychlik (2004) except for the assumption that $h(0)=0$. But careful analysis of the proof of Lemma 3 of Danielak and Rychlik (2004) shows that it holds true under less restrictive condition $h(0) \geq 0$ as well. By Lemma 1 we see that the function $\hat{h}_{*, r}=1-\hat{f}_{*, r}$ satisfies the conditions (A) for $r \geq 2$ and $\gamma_{r}>1+\alpha$ if $\alpha>0$, or $\gamma_{r}>1$ if $\alpha \leq 0$.

Note that each function $h$ satisfying the conditions (A) has the unique minimum $h(a)<0$, so it has the unique zero $\theta \in(a, d)$. By Lemma 4(ii) of Danielak and Rychlik (2004), if

$$
H(x)=\int_{0}^{x} h(u) w(u) d u, \quad x \in(0, d),
$$

then the equation

$$
H(\gamma)=W(\gamma) h(\gamma)
$$

has exactly one solution $\gamma \in(0, d)$, which belongs to the interval $(a, \theta)$.

The precise form of $P_{W} h$ for $h$ satisfying (A) is determined by the following auxiliary functions 


$$
\begin{aligned}
\lambda_{W}(y) & =\frac{\int_{y}^{d}(x-y)(h(x)-h(y)) w(x) d x}{\int_{y}^{d}(x-y)^{2} w(x) d x}, \\
K_{W}(y) & =\lambda_{W}(y)-h^{\prime}(y), \\
L_{W}(y) & =\int_{y}^{d}\left[h(x)-h(y)-\lambda_{W}(y)(x-y)\right] w(x) d x .
\end{aligned}
$$

The following results is now easy extension of Propostion 1 of Danielak and Rychlik (2004).

Proposition 2 Assume that $\gamma$ is given by (11) and let

$$
\mathscr{K}=\left\{\gamma<y<b: K_{W}(y) \geq 0 \text { and } L_{W}(y)=0\right\} .
$$

If $\mathscr{K} \neq \varnothing$ and $y^{*}=\sup \mathscr{K}$, then

$$
P_{W} h(x)= \begin{cases}h(\gamma), & \text { for } 0 \leq x \leq \gamma \\ h(x), & \text { for } \gamma<x \leq y^{*} \\ h\left(y^{*}\right)+\lambda^{*}\left(x-y^{*}\right), & \text { for } y^{*}<x<d\end{cases}
$$

where $\lambda^{*}=\lambda_{W}\left(y^{*}\right)$. Otherwise, if $\mathscr{K}=\varnothing$, then

$$
P_{W} h(x)=-\frac{H(y)}{W(y)}\left[\frac{(x-y) \mathbf{1}_{[y, d)}(x)}{\int_{y}^{d}(x-y) w(x) d x}-1\right]
$$

for the greatest $y \in(0, \gamma]$ satisfying

$$
\frac{\int_{y}^{d} h(x) w(x) d x}{\int_{0}^{y} w(x) d x}=\frac{\int_{y}^{d}(x-y) w(x) d x \int_{y}^{d}(x-y) h(x) w(x) d x}{\int_{y}^{d}(x-y)^{2} w(x) d x-\left(\int_{y}^{d}(x-y) w(x) d x\right)^{2}} .
$$

\section{Main results}

We are now ready to formulate the main results, concerning the family of the DGFR $(\alpha)$ distributions, i.e., for $W=W_{\alpha}$. In order to fulfil the condition (6) we need to assume that $\alpha>-\frac{1}{2}$. In the formulation and the proof of the main result we use the following notation and auxiliary functions. Firstly, for $1 \leq j \leq r$ we define

$$
\sigma_{j, r}(\alpha)= \begin{cases}\frac{1}{\alpha}\left(1-\prod_{i=j}^{r} \frac{\gamma_{i}}{\gamma_{i}+\alpha}\right), & \text { if } \alpha \neq 0, \\ \sum_{i=j}^{r} \frac{1}{\gamma_{i}}, & \text { if } \alpha=0 .\end{cases}
$$


Then $\sigma_{j, r}(\alpha)>0$ for $j=1, \ldots, r$, if $\gamma_{r} \geq 1$ and $\alpha>-\frac{1}{2}$. Also, $\sigma_{j, r}(\alpha) \rightarrow \sigma_{j, r}(0)$ if $\alpha \rightarrow 0$. It is easy to verify that the sequence $\sigma_{j, r}(\alpha), 1 \leq j \leq r$, is decreasing with respect to $j$. Moreover, we define auxiliary functions which are used in the statement and the proof of the main result. First, let

$$
\lambda_{\alpha}(y)=\frac{(1+\alpha)(1+2 \alpha)}{2(1-\alpha y)}\left\{-\sum_{j=1}^{r-1} \frac{\sigma_{j, r}(\alpha)}{\gamma_{j}} \hat{f}_{*, j}(y)+\left(\frac{1}{1+\alpha}-\frac{\sigma_{r, r}(\alpha)}{\gamma_{r}}\right) \hat{f}_{*, r}(y)\right\} .
$$

Moreover, we define

$$
K_{\alpha}(y)=\frac{1}{1-\alpha y} \sum_{j=1}^{r} b_{j} \hat{f}_{*, j}(y)
$$

where

$$
\begin{aligned}
b_{j} & =-\frac{(1+\alpha)(1+2 \alpha)}{2 \gamma_{j}} \sigma_{j, r}(\alpha), \quad 1 \leq j \leq r-2, \\
b_{r-1} & =-\frac{(1+\alpha)(1+2 \alpha)}{2 \gamma_{r-1}} \sigma_{r-1, r}(\alpha)+\gamma_{r}, \\
b_{r} & =-\frac{\left(\gamma_{r}-1\right)\left(\gamma_{r}+\left(\alpha+\frac{1}{2}\right)\right)\left(\gamma_{r}-(1+\alpha)\right)}{\gamma_{r}\left(\gamma_{r}+\alpha\right)},
\end{aligned}
$$

and

$$
L_{\alpha}(y)=\frac{1}{1-W_{\alpha}(y)} \sum_{j=1}^{r} d_{j} \hat{f}_{*, j}(y)
$$

where

$$
\begin{aligned}
d_{j} & =\frac{1}{\gamma_{j}}\left(\frac{1+2 \alpha}{2} \sigma_{j, r}(\alpha)-1\right), \quad 1 \leq j \leq r-1, \\
d_{r} & =\frac{\left(\gamma_{r}-1\right)\left(\gamma_{r}-(1+\alpha)\right)}{2(1+\alpha) \gamma_{r}\left(\gamma_{r}+\alpha\right)}
\end{aligned}
$$

Proposition 3 Assume that $\alpha>-\frac{1}{2}$ and let $X_{\gamma}^{(r)}$ with $r \geq 2$ be the generalized order statistic based on the distribution function $F \in D G F R(\alpha)$ and parameters $\gamma=\left(\gamma_{1}, \ldots, \gamma_{r}\right)$, where $\gamma_{1} \geq \gamma_{2} \geq \cdots \geq \gamma_{r}>1+\max (\alpha, 0)$. Let $\delta \in\left(W_{\alpha}(a), 1\right)$ be the unique solution to

$$
(1-\delta) \sum_{j=1}^{r} \frac{1}{\gamma_{j}} f_{*, j}(\delta)=1-\delta f_{*, r}(\delta)
$$

and let $\gamma=W_{\alpha}^{-1}(\delta)$. 
Let $v$ be the only solution to equation $K_{\alpha}(v)=0$ in the interval $(a, b)$. If the condition

$$
K_{\alpha}(\gamma)>0 \text { and } L_{\alpha}(\gamma)<0<L_{\alpha}(v)
$$

holds true, then there exists the unique $y \in(\gamma, v)$ such that $L_{\alpha}(y)=0$ and we have the following bound

$$
\mathbb{E} \frac{X_{\gamma}^{(r)}-\mu}{\sigma} \geq-B_{1}
$$

where

$$
\begin{aligned}
B_{1}^{2}= & \delta\left(1-f_{*, r}(\delta)\right)^{2}+\int_{\delta}^{W(y)}\left(1-f_{*, r}(x)\right)^{2} d x \\
& +\left(1-W_{\alpha}(y)\right)\left[\left(\hat{h}_{*, r}(y)\right)^{2}+2 \lambda \hat{h}_{*, r}(y) \frac{1-\alpha y}{1+\alpha}+\frac{2 \lambda^{2}(1-\alpha y)^{2}}{(1+\alpha)(1+2 \alpha)}\right],
\end{aligned}
$$

and $\lambda=\lambda_{\alpha}(y)$. The equality in (21) is attained for $F$ satisfying

$$
F^{-1}\left(W_{\alpha}(x)\right)= \begin{cases}\mu+\frac{\sigma}{B_{1}}\left(1-\hat{f}_{*, r}(\gamma)\right), & \text { for } 0 \leq x<\gamma \\ \mu+\frac{\sigma}{B_{1}}\left(1-\hat{f}_{*, r}(x)\right), & \text { for } \gamma \leq x<y \\ \mu+\frac{\sigma}{B_{1}}\left[1-\hat{f}_{*, r}(y)+\lambda(x-y)\right], & \text { for } y \leq x<d\end{cases}
$$

Otherwise, i.e., if (20) fails, then we define $y \in(0, \gamma]$ as the greatest solution to equation

$$
1-\sum_{j=1}^{r} \frac{1}{\gamma_{j}} \hat{f}_{*, j}(y)=(1+2 \alpha) W_{\alpha}(y) \sum_{j=1}^{r} \frac{1}{\gamma_{j}}\left[1+(1+\alpha) \sigma_{j, r}(\alpha)\right] \hat{f}_{*, j}(y)
$$

and we put $\rho=W_{\alpha}(y), \lambda=\lambda_{\alpha}(y)$. Then we have the following bound

$$
\mathbb{E} \frac{X_{\gamma}^{(r)}-\mu}{\sigma} \geq-B_{2}
$$

where

$$
B_{2}=\sqrt{\frac{1+2 \alpha \rho-(1+2 \alpha) \rho^{2}}{(1+2 \alpha) \rho^{2}}}\left(1-\sum_{j=1}^{r} \frac{1}{\gamma_{j}} \hat{f}_{*, j}(y)\right) .
$$

The equality in (24) holds for F such that

$$
F^{-1}\left(W_{\alpha}(x)\right)= \begin{cases}\mu+\frac{\sigma}{B_{2}}\left(1-\hat{f}_{*, r}(y)\right), & \text { for } 0 \leq x<y \\ \mu+\frac{\sigma}{B_{2}}\left[1-\hat{f}_{*, r}(y)+\lambda(x-y)\right], & \text { for } y \leq x<d\end{cases}
$$


Proof It suffices to determine $P_{W_{\alpha}} \hat{h}_{*, r}$ applying Proposition 2 with $W=W_{\alpha}, h=\hat{h}_{*, r}$ and $H=\hat{H}_{*, r}$ defined by

$$
\hat{H}_{*, r}(y)=\int_{0}^{y} \hat{h}_{*, r}(t) w_{\alpha}(t) d t .
$$

First we determine $\gamma$ defined by (11). Since $\hat{h}_{*, r}=1-\hat{f}_{*, r}$, then (11) is equivalent to $F_{*, r}\left(W_{\alpha}(\gamma)\right)=W_{\alpha}(\gamma) \hat{f}_{*, r}(\gamma)$ or to (5) with $\delta=W_{\alpha}(\gamma)$. Using the representation (12) of Bieniek (2008b)

$$
1-F_{*, r}(u)=(1-u) \sum_{j=1}^{r} \frac{1}{\gamma_{j}} f_{*, j}(u)
$$

we get (19). Note that an easy application of the last formula shows that

$$
\hat{H}_{*, r}(y)=(1-W(y))\left(\sum_{j=1}^{r} \frac{1}{\gamma_{j}} \hat{f}_{*, j}(y)-1\right) .
$$

Next we determine the conditions which ensure that $P_{W_{\alpha}} \hat{h}_{*, r}$ is of the form (13). To this aim we need to study the properties of the functions $\lambda_{\alpha}=\lambda_{W_{\alpha}}$, $K_{\alpha}=K_{W_{\alpha}}$ and $L_{\alpha}=L_{W_{\alpha}}$ given by (12). Writing temporarily $\lambda_{W}(y)=$ $\lambda_{W}(y ; h), K_{W}(y)=K_{W}(y ; h)$ and $L_{W}(y)=L_{W}(y ; h)$, we easily obtain that $\lambda_{W_{\alpha}}\left(y ; \hat{h}_{*, r}\right)=-\lambda_{W_{\alpha}}\left(y ; \hat{f}_{*, r}\right)$ and $K_{W_{\alpha}}\left(y ; \hat{h}_{*, r}\right)=-K_{W_{\alpha}}\left(y ; \hat{f}_{*, r}\right)$ and $L_{W_{\alpha}}\left(y ; \hat{h}_{*, r}\right)=-L_{W_{\alpha}}\left(y ; \hat{f}_{*, r}\right)$. The functions on the right hand sides of the last three equalities are determined in (A.1), (3.2) and (3.3) of Bieniek (2008a). This easily implies the representation of $\lambda_{\alpha}, K_{\alpha}$ and $L_{\alpha}$ given in (16), (17) and (18).

The next step is to determine the zeros of $K_{\alpha}$ and $L_{\alpha}$ in order to determine when the set $\mathscr{K}$ is not empty. First we determine the zeros of (17). Note that since $\lambda_{\alpha}(y)$ is the slope of the best linear approximation of $\hat{h}_{*, r}$ restricted to the interval $(y, d)$ and passing through $\left(y, \hat{h}_{*, r}(y)\right)$, then easy geometric considerations show that $K_{\alpha}(a)>$ $0>K_{\alpha}(b)$. Since $b_{j}<0, j=1, \ldots, r-2$ and $b_{r}<0$, we conclude that function $K_{\alpha}$ is either - or -+- on $(0, d)$. The first situation is not possible since $K(a)>0$, therefore $K$ is negative, positive and ultimately negative on $(0, d)$ and hence positive and negative on $(a, b)$. Let $v$ denote the only solution of the equation $K_{\alpha}(v)=0$ in $(a, b)$. So if $K_{\alpha}(\gamma)>0$, then $\mathscr{K}^{+}=\left\{y \in(\gamma, b): K_{\alpha}(y)>0\right\}=(\gamma, v)$. Otherwise, if $K_{\alpha}(\gamma) \leq 0$, then $\mathscr{K}^{+}=\varnothing$ and in consequence $\mathscr{K}=\varnothing$.

Now we analyze the function (18). Note that $d_{r}>0$. Moreover, consider $\delta_{j}=\gamma_{j} d_{j}$, $j=1, \ldots, r-1$. It is easily seen that $\delta_{1} \geq \cdots \geq \delta_{r-1}$. This implies that $d_{1}, \ldots, d_{r-1}$ are either all positive or all negative, or first positive, then negative. Therefore $L_{\alpha}$ is either + or -+ or +-+ on $(0, d)$. By Lemma 4 of Gajek and Rychlik (1998) (see also Lemma 7 of Danielak and Rychlik (2004)) we get that $L_{\alpha}$ is either + or -+ on $\mathscr{K}^{+}=(\gamma, v)$ provided that this set is non-empty.

Summing up if $K_{\alpha}(\gamma)>0$ and $L_{\alpha}(\gamma)<0<L_{\alpha}(v)$, then $\mathscr{K}=\{y\}$, where $y \in(\gamma, v)$ is the unique solution to $L_{\alpha}(y)=0$ in $(\gamma, v)$. Therefore if (20) holds, 
then $P_{W \alpha} \hat{h}_{*, r}$ is of the form (13) with $y^{*}=y$ and $\lambda^{*}=\lambda_{\alpha}(y)$. The value of the corresponding bound $B_{1}$ given by (21) is just $\mathscr{L}_{W_{\alpha}}^{2}-$ norm of $P_{W_{\alpha}} \hat{h}_{*, r}$, and (22) follows from (9).

On the other hand if (20) fails, then $\mathscr{K}=\varnothing$ and $P_{W_{\alpha}} \hat{h}_{*, r}$ is of the form (14). The parameter $y$ is defined by (15) which is equivalent to (23). The value of the bound (24) is just the $\mathscr{L}_{W_{\alpha}}^{2}$-norm of (14) with $W=W_{\alpha}$ which amounts to

$$
-\frac{\hat{H}_{*, r}(y)}{W_{\alpha}(y)} \sqrt{\frac{1+(1+2 \alpha) W_{\alpha}(y)}{(1+2 \alpha)\left(1-W_{\alpha}\right)(y)}} .
$$

Using (26) we transform this into the right hand side of (25). Again, the formula for $F$ for which the bound is attained follows from (9), which completes the proof.

\subsection{Remaining cases}

If $\alpha>0$ and $1<\gamma_{r} \leq 1+\alpha$, then according to Lemma 1 the function $\hat{h}_{*, r}$ is first decreasing and then convex increasing. Its projection $P^{\nearrow} \hat{h}_{*, r}$ onto the convex cone of nondecreasing functions in $\mathscr{L}_{W}^{2}$ is determined by Moriguti approach. Thus

$$
P^{\nearrow} \hat{h}_{*, r}(u)= \begin{cases}\hat{h}_{*, r}(\gamma), & \text { for } 0 \leq u \leq \gamma \\ \hat{h}_{*, r}(u), & \text { for } \gamma \leq u \leq d\end{cases}
$$

where $\gamma=W_{\alpha}^{-1}(\delta)$, and $\delta$ is the unique solution to (19). Therefore, $P^{\nearrow} \hat{h}_{*, r} \in \mathscr{C}_{W_{\alpha}}$ and $P_{W_{\alpha}} \hat{h}_{*, r}=P^{\nearrow} \hat{h}_{*, r}$. This implies the bound

$$
\mathbb{E} \frac{X_{\gamma}^{(r)}-\mu}{\sigma} \geq-B_{3},
$$

where

$$
\begin{aligned}
B_{3}^{2} & =W_{\alpha}(\gamma) \hat{h}_{*, r}^{2}(\gamma)+\int_{\gamma}^{d}\left(\hat{h}_{*, r}(u)\right)^{2} w_{\alpha}(u) d u \\
& =\delta\left(h_{*, r}(\delta)\right)^{2}+\int_{\delta}^{1}\left(h_{*, r}(u)\right)^{2} d u
\end{aligned}
$$

which does not depend on $\alpha$. The equality in (27) holds for $F$ satisfying

$$
F(x)= \begin{cases}0, & \text { if } \frac{x-\mu}{\sigma}<\frac{h_{*, r}(\delta)}{B_{3}}, \\ h_{*, r}^{-1}\left(B_{3} \frac{x-\mu}{\sigma}\right), & \text { if } \frac{h_{*, r}(\delta)}{B_{3}} \leq \frac{x-\mu}{\sigma}<\frac{1}{B_{3}}, \\ 1, & \text { if } \frac{x-\mu}{\sigma} \geq \frac{1}{B_{3}}\end{cases}
$$

Finally, if $\gamma_{r} \leq 1$, then $\hat{h}_{*, r}$ is decreasing, so $P_{W_{\alpha}} \hat{h}_{*, r}$ is constant and the lower bound is just 0 , so $E X_{\gamma}^{(r)} \geq \mu$. 
Table 1 Bounds on the expectations of the standardized progressively type II censored order statistics for $R_{1}=(1,2,3,4,5,6,7)$

\begin{tabular}{|c|c|c|c|c|c|c|c|c|c|}
\hline \multirow[b]{2}{*}{$r$} & \multicolumn{3}{|l|}{ General $F$} & \multicolumn{3}{|l|}{$F \in \mathrm{DD}$} & \multicolumn{3}{|l|}{$\underline{F \in \text { DFR }}$} \\
\hline & Lower & Upper & Range & Lower & Upper & Range & Lower & Upper & Range \\
\hline 2 & -2.7136 & 0.0171 & 2.7307 & -1.5369 & 0 & 1.5369 & -0.9411 & 0 & 0.9411 \\
\hline 3 & -2.1742 & 0.0665 & 2.2407 & -1.4345 & 0 & 1.4345 & -0.9080 & 0 & 0.9080 \\
\hline 4 & -1.8259 & 0.1197 & 1.9456 & -1.3288 & 0 & 1.3288 & -0.8708 & 0 & 0.8708 \\
\hline 5 & -1.5481 & 0.1749 & 1.7230 & -1.2150 & 0 & 1.2150 & -0.8266 & 0 & 0.8266 \\
\hline 6 & -1.2840 & 0.2367 & 1.5207 & -1.0797 & 0 & 1.0797 & -0.7678 & 0 & 0.7678 \\
\hline 7 & -0.9546 & 0,3191 & 1.2737 & -0.8712 & 0 & 0.8712 & -0.6643 & 0 & 0.6643 \\
\hline
\end{tabular}

\section{Applications}

We illustrate the above results via one of the particular examples of the gOSs, which is the model of the progressively type II censored order statistics. Such data often occur, e.g., in life testing or reliability studies. The censored life testing, although is not as efficient as life testing under the complete sample, is usually implemented in case when some resources saving, i.e., time, is our priority. In such reliability tests a fixed number of components is placed in test at the same time, and we decide to terminate the test earlier (e.g., due to the long life of certain components) and perform the reliability analysis based on the observed data up to the time of termination. The test is terminated when a predetermined number of component failures have occurred, while the times to failure of each failed unit are recorded.

Precisely, in the type II censoring scheme, we consider $N$ independent lifetimes $X_{1}, \ldots, X_{N}$ based on the common distribution function $F$, and the experiment where particular amount of surviving units are removed at various experiment stages. After the $i$-th failure, $R_{i}$ still living units are removed at random, $i=1, \ldots, n$. Formally, we observe $n \leq N$ failures $X_{*, \gamma}^{(1)}, \ldots, X_{*, \gamma}^{(n)}$, according to the fixed censoring scheme $R=\left(R_{1}, \ldots, R_{n}\right)$ for non-negative integers $R_{1}, \ldots, R_{n}$ with $N=n+\sum_{i=1}^{n} R_{i}$. In this case we have $\gamma_{j}=N+1-j-\sum_{i=1}^{j-1} R_{i}, j=1, \ldots, n$ and $\gamma_{1}=N$.

It may be of the interest to consider the time to failure of the $r$ th item as the population mean $\mu$ estimator, in terms of the range of its bias. In order to do that, we need both upper and lower bounds of the expected standardized progressively type II censored order statistics $E \frac{X_{*, \gamma}^{(r)}-\mu}{\sigma}$, possibly in various cases of the initial distribution function. In this paper we have considered $N=35, n=7$ and the following three censoring schemes: $R_{1}=(1,2,3,4,5,6,7), R_{2}=(4,4,4,4,4,4,4), R_{3}=(7,6,5,4,3,2,1)$ (Bieniek 2016a, cf.) in the numerical calculations. The tables below present the lower and the upper bounds for three various cases of the base distribution function $F$ : general case without any restrictions, decreasing density and decreasing failure rate families for these particular censoring schemes. We also provide the spread of the bounds, denoted in tables as range. 
Table 2 Bounds on the expectations of the standardized progressively type II censored order statistics for $R_{2}=(4,4,4,4,4,4,4)$

\begin{tabular}{|c|c|c|c|c|c|c|c|c|c|}
\hline \multirow[b]{2}{*}{$r$} & \multicolumn{3}{|l|}{ General $F$} & \multicolumn{3}{|l|}{$\underline{F \in \mathrm{DD}}$} & \multicolumn{3}{|l|}{$\underline{F \in \mathrm{DFR}}$} \\
\hline & Lower & Upper & Range & Lower & Upper & Range & Lower & Upper & Range \\
\hline 2 & -2.6405 & 0.0180 & 2.6585 & -1.5273 & 0 & 1.5273 & -0.9381 & 0 & 0.9381 \\
\hline 3 & -2.0529 & 0.0715 & 2.1244 & -1.4060 & 0 & 1.4060 & -0.8984 & 0 & 0.8984 \\
\hline 4 & -1.6659 & 0.1318 & 1.7977 & -1.2733 & 0 & 1.2733 & -0.8503 & 0 & 0.8503 \\
\hline 5 & -1.3546 & 0.1976 & 1.5522 & -1.1251 & 0 & 1.1251 & -0.7893 & 0 & 0.7893 \\
\hline 6 & -1.0600 & 0.2753 & 1.3353 & -0.9459 & 0 & 0.9459 & -0.7042 & 0 & 0.7042 \\
\hline 7 & -0.7009 & 0.3858 & 1.0867 & -0.6731 & 0 & 0.6731 & -0.5489 & 0 & 0.5489 \\
\hline
\end{tabular}

Table 3 Bounds on the expectations of the standardized progressively type II censored order statistics for $R_{3}=(7,6,5,4,3,2,1)$

\begin{tabular}{|c|c|c|c|c|c|c|c|c|c|}
\hline \multirow[b]{2}{*}{$r$} & \multicolumn{3}{|l|}{ General $F$} & \multicolumn{3}{|l|}{$\underline{F \in \mathrm{DD}}$} & \multicolumn{3}{|l|}{$F \in \mathrm{DFR}$} \\
\hline & Lower & Upper & Range & Lower & Upper & Range & Lower & Upper & Range \\
\hline 2 & -2.5580 & 0.0190 & 2.5770 & -1.5157 & 0 & 1.5157 & -0.9344 & 0 & 0.9344 \\
\hline 3 & -1.9061 & 0.0779 & 1.9840 & -1.3667 & 0 & 1.3667 & -0.8848 & 0 & 0.8848 \\
\hline 4 & -1.4575 & 0.1494 & 1.6069 & -1.1868 & 0 & 1.1869 & -0.8165 & 0 & 0.8165 \\
\hline 5 & -1.0818 & 0.2355 & 1.3173 & -0.9680 & 0 & 0.9680 & -0.7174 & 0 & 0.7174 \\
\hline 6 & -0.7127 & 0.3516 & 1.0643 & -0.6850 & 0 & 0.6850 & -0.5581 & 0 & 0.5581 \\
\hline 7 & -0.2450 & 0.5601 & 0.8051 & -0.2450 & 0.2887 & 0.5337 & -0.2295 & 0 & 0.2295 \\
\hline
\end{tabular}

It would seem, that the scheme $R_{1}$ preserves the most information among all considered schemes, hence it should provide the best possible approximation of the population mean $\mu$ in this particular setting of the progressively type II censored order statistics. The intuition is that it is better to leave the items in the experiment as long as possible, like the scheme $R_{1}$ does. However, in this specific case, the better approximation is delivered by the scheme $R_{3}$, which is in some way against the intuition. One can suspect that the similar situation holds for other values of $N, n$, and censoring schemes $R$. Unfortunately, this supposition cannot be proved in the general case, since the estimation formulas are too complicated (Tables 1,2,3).

Acknowledgements The authors are greatly indebted to anonymous reviewers for many valuable comments which helped in the preparation of the final version of the paper. This work was supported by Polish National Science Centre under Grant No. 2015/19/B/ST1/03100.

Open Access This article is distributed under the terms of the Creative Commons Attribution 4.0 International License (http://creativecommons.org/licenses/by/4.0/), which permits unrestricted use, distribution, and reproduction in any medium, provided you give appropriate credit to the original author(s) and the source, provide a link to the Creative Commons license, and indicate if changes were made. 


\section{References}

Barakat HM, Nigm EM, El-Adll ME, Yusuf M (2016) Prediction of future generalized order statistics based on exponential. Distribution with random sample size. Stat Pap. https://doi.org/10.1007/s00362-0160779-2

Barlow RE, van Zwet WR (1970) Asymptotic properties of isotonic estimators for the generalized failure rate function. I. Strong consistency. In: Nonparametric techniques in statistical inference (Proc. Sympos., Indiana Univ., Bloomington, Ind., 1969). Cambridge University Press, London, pp 159-176

Belzunce F, Martínez-Riquelme C (2015) Some results for the comparison of generalized order statistics in the total time on test and excess wealth orders. Stat Pap 56:1175-1190

Bieniek M (2006) Projection bounds on expectations of generalized order statistics from DFR and DFRA families. Statistics 40:339-351

Bieniek M (2007a) Projection mean-variance bounds on expectations of kth record values from restricted families. Commun Stat Theory Methods 36(4):679-692

Bieniek M (2007b) Variation diminishing property of densities of uniform generalized order statistics. Metrika 65:297-309

Bieniek M (2008a) On families of distributions for which optimal bounds on expectations of GOS can be derived. Commun Stat Theory Methods 37:1997-2009

Bieniek M (2008b) Projection bounds on expectations of generalized order statistics from DD and DDA families. J Stat Plan Inference 138:971-981

Bieniek M (2016a) Sharp bounds for the bias of trimmed means of progressively censored order statistics. Probab Math Stat 36(1):99-112

Bieniek M (2016b) Sharp bounds on the bias of trimean. Stat Pap 57:365-379

Cramer E, Kamps U (2003) Marginal distributions of sequential and generalized order statistics. Metrika 58:293-310

Cramer E, Kamps U, Rychlik T (2002) Evaluations of expected generalized order statistics in various scale units. Appl Math (Warsaw) 29(3):285-295

Cramer E, Kamps U, Rychlik T (2004) Unimodality of uniform generalized order statistics, with applications to mean bounds. Ann Inst Stat Math 56(1):183-192

Danielak K, Rychlik T (2004) Sharp bounds for expectations of spacings from decreasing density and failure rate families. Appl Math (Warsaw) 31:369-395

Gajek L, Rychlik T (1998) Projection method for moment bounds on order statistics from restricted families. II. Independent case. J Multivar Anal 64:156-182

Goroncy A (2014) Bounds on expected generalized order statistics. Statistics 48(3):593-608

Goroncy A (2017) Upper non-positive bounds on expectations of generalized order statistics from DD and DDA populations. Commun Stat Theory Methods 46(24):11972-11987

Kamps U (1995a) A concept of generalized order statistics. B. G Teubner, Stuttgart

Kamps U (1995b) A concept of generalized order statistics. J Stat Plan Inference 48:1-23

Mahmoud MAW, Al-Nagar HS (2009) On generalized order statistics from linear exponential distribution and its characterization. Stat Pap 50:407-418

Mathai AM (1993) A handbook of generalized special functions for statistical and physical sciences. Oxford Science Publications, New York

Moriguti S (1953) A modification of Schwarz's inequality with applications to distributions. Ann Math Stat 24:107-113

Okolewski A, Kaluszka M (2008) Bounds for expectations of concomitants. Stat Pap 49:603-618

Rychlik T (2001) Projecting statistical functionals. Springer, New York

Rychlik T (2010) Evaluations of generalized order statistics from bounded populations. Stat Pap 51:165-177 